\title{
Guidelines for authors who would like to submit their figures electronically to AMS
}

It is now possible for the AMS to take advantage, in many cases, of the electronic files used by authors to create computergenerated figures directly in the typesetting process. Using these files can often lead to a higher quality of reproduction in the journals and represents an efficient use of the electronic medium. The following serve as guidelines for electronic submission of figures. Authors should feel free to contact the technical editors at AMS Headquarters if they have questions.

\section{General guidelines:}

1. Authors should not submit the electronic files containing their figures with their initial submission. Instead they should include the electronic files with the final revised manuscript so that they can be forwarded to AMS Headquarters with the manuscript by the editor.

2. Authors should only submit their files in electronic form if their figures were created that way to begin with-that is, authors should not submit scanned images of hardcopy figures because the AMS typesetters can, in general, process those hardcopy figures in ways that optimize their reproduction in the printed journal. Also, figures created with vector graphics should be submitted as files with the vector information rather than as bitmapped image files.

3. Authors should always supply high-quality originals of all their figures, including those being submitted electronically, so that the hardcopy can be used if the electronic files prove to be unusable.

4. As with all figures, authors should take care to consider the final size and layout of the figure. Most figures will be reproduced at either column width $(7.9 \mathrm{~cm}, 3.125 \mathrm{in}$.) or double-column width $(16.5 \mathrm{~cm}, 6.5 \mathrm{in}$.). The electronic file should contain the figure at a size as close to final reproduction size as possible. All text, including axis labels, contour labels, and symbols, should be sized so that it will be easily readable at the final reproduction size, and figures should avoid use of text of very different size within the same figure.

5. Authors should make sure that the electronic representation of the figure is closely cropped both vertically and horizontally. That is, the electronic representation should contain as little white space around the figure as possible.

6. Authors should indicate the format, operating system, program, and version number of the software used to create the figure on the diskette (or other media) containing the figure.

7. Each figure should be saved as a separate file with a logical name that will help the typesetter identify the correct file for each figure.

8. When submitting electronic color images, indicate the color file format used and include a four-color proof of sufficiently high quality to serve as an original if necessary.

9. While uncompressed files are desirable, the following compression software can be used to save the images onto transportable media: Stuffit or Stuffit Delux, PKZip, Aladdan, DiskDoubler, or a self-extracting archive. Indicate the compression program used and its version number with the submission.

10. If the graphics software used allows compression at the time of output as an EPS file, this option should not be used. Instead, output the file without compression and then, if necessary, use a separate compression routine on this file.

\section{Acceptable submission standards:}

Line art should be submitted in encapsulated PostScript (EPS), in either vector or bitmapped form, or TIFF, with bitmapped figures having 1000-1600 dot per inch (dpi) resolution at the size the figure will be printed. Additionally, AMS can accept the actual application files from the program used to create the figure if it was created in a Macintosh or Windows environment with Adobe Photoshop, Adobe Illustrator, Aldus Freehand, or Corel Draw.

Black-and-white halftones should be submitted in EPS or TIFF.

Color figures should be submitted in CMYK EPS, DCS, or CMYK TIFF. RGB EPS and RGB TIFF do not reproduce well and should be avoided.

Note that PostScript files, such as those generated by the NCAR Graphics translator, that are not encapsulated PostScript are not acceptable. PostScript files can often be imported into another graphics utility, such as Adobe Photoshop, and resaved as an EPS file, which can then be submitted to AMS.

\section{Acceptable media:}

The AMS is not yet able to accept graphic files via the Internet, so all electronic figure submissions must use some form of physical media. Figure files can be submitted on any form of DOS diskette, Mac diskette (3.5" low or high density), SyQuest (44 or $88 \mathrm{MB}$ ), Bernoulli $90 \mathrm{MB}, 8 \mathrm{~mm}$ Unix-format tar (2.3 or $5 \mathrm{~GB}$ ), Q150 cartridges (Unix tar), or Canon Magneto-Optical $(600 \mathrm{MB})$. Be sure that the media matches the operating system and platform used to create the file (for example, do not submit a figure file originally generated with Windows software on a cartridge written with a Unix machine). 